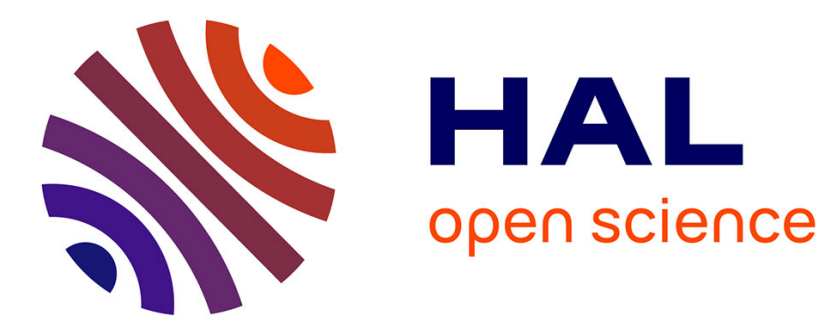

\title{
Evaluation of the Parameters of a Constitutive Mode1 for b.c.c. Metals Based on Thermal Activation
}

\author{
F. Buy, J. Farre, J. Klepaczko, G. Talabart
}

\section{To cite this version:}

F. Buy, J. Farre, J. Klepaczko, G. Talabart. Evaluation of the Parameters of a Constitutive Mode1 for b.c.c. Metals Based on Thermal Activation. Journal de Physique IV Proceedings, 1997, 07 (C3), pp.C3-631-C3-636. 10.1051/jp4:19973108 . jpa-00255392

\section{HAL Id: jpa-00255392 https://hal.science/jpa-00255392}

Submitted on 1 Jan 1997

HAL is a multi-disciplinary open access archive for the deposit and dissemination of scientific research documents, whether they are published or not. The documents may come from teaching and research institutions in France or abroad, or from public or private research centers.
L'archive ouverte pluridisciplinaire HAL, est destinée au dépôt et à la diffusion de documents scientifiques de niveau recherche, publiés ou non, émanant des établissements d'enseignement et de recherche français ou étrangers, des laboratoires publics ou privés. 


\title{
Evaluation of the Parameters of a Constitutive Model for b.c.c. Metals Based on Thermal Activation
}

\author{
F. Buy, J. Farre, J.R. Klepaczko* and G. Talabart \\ Commissariat à l'Énergie Atomique, Centre de Valduc, BP.14, 21120 Is-sur-Tille, France \\ * LPMM/ISGMP, Université de Metz, Ile de Saulcy, 57000 Metz, France
}

\begin{abstract}
Résumé : L'objet de cet article consiste à modéliser le comportement mécanique d'un tantale et à travers celui-ci des métaux à structure cubique centrée en se basant sur un formalisme physique proposé par J.R. Klepaczko[1]. La gamme de vitesse de déformation étudiée est comprise entre $10^{-4}$ et $10^{3} s^{-1}$. Nous présentons ici une procédure expérimentale et analytique qui permet de déterminer les paramètres du modèle. A partir du modèle ainsi déterminé, nous simulons, en bon accord avec les résultats expérimentaux, des essais à vitesse constante ainsi que des sauts de vitesse. Une discussion sur les micro-mécanismes de déformation permet une validation théorique des coefficients du modèle.
\end{abstract}

\begin{abstract}
Absract: The aim of this paper consists in modelling the mechanical behaviour of tantalum as an example for b.c.c. metals. The range of strain rates is between $10^{-4}$ et $10^{3} \mathrm{~s}^{-1}$. A formalism proposed by J.R. Klepaczko [1] and based on physical relations has been adopted. A methodology based on experiments and analysis is presented, which enables to determine the parameters. Tests at constant strain rates and jump tests are simulated showing a good agreement with the experiments. A discussion on deformation mechanisms gives a theoretical validation of the model's coefficients.
\end{abstract}

\section{INTRODUCTION}

La contrainte d'écoulement des métaux polycristallins présente une sensibilité importante à la température $T$ et à la vitesse de déformation $\dot{\gamma}$. Les contributions de ces paramètres - dits de chargement- ont fait l'objet de nombreuses études[2, 3].

Plus récemment, il a été mis en évidence que l'évolution de ces paramètres au cours du temps joue un rôle sur la réponse mécanique du métal considéré[1]. Une fois qu'un métal entre dans le domaine plastique, il se consolide lorsque la déformation augmente. Cet écrouissage est relié à l'évolution de son état microstructural (densité de dislocations, taille de grains, macles, texture...). Dans la plupart des modèles de loi de comportement, le seul paramètre traduisant l'effet de l'écrouissage est le taux de déformation plastique $\gamma$. Cependant, il a été mis en évidence que ce taux ne peut être considéré comme une variable représentant l'état du matériau. C'est pourquoi nous adoptons un formalisme différent qui permet de tenir compte des effets $d$ 'histoire du chargement.

Dans la suite de l'étude, nous assimilons l'évolution de l'état microstructural à celle de la densité de dislocations $\varrho$ au sein du matériau, et ne prenons pas en considération la taille de grains, la texture et l'apparition de cellules de dislocations à l'origine de la formation de sous-grains. Cette simplification, bien que restrictive, nous permet d'appréhender certains phénomènes et de retranscrire des observations faites lors d'essais de saut de vitesse sur une nuance de tantale polycristallin.

\section{MODELE}

Nous avons choisi de modéliser le comportement du tantale en nous appuyant sur le formalisme proposé par Klepaczko[1, 4,5] qui repose sur la théorie de l'activation thermique. Ce modèle comporte deux volets principaux:

- des lois cinétiques relient la contrainte d'écoulement aux paramètres de chargement et à l'état microstructural du matériau et

- des lois d'évolution rendent compte de la modification des défauts microstructuraux tout au long de la déformation. 


\subsection{Lois cinétiques}

Le cadre de notre étude se limite à des vitesses de déformation $\dot{\gamma}$ comprises entre $10^{-4}$ et $10^{3} \mathrm{~s}^{-1}$ Les essais de cisaillement ont été effectués à température $T$ ambiante, ce qui dans le cas du tantale correspond à un dixième de la température de fusion. Sous ces conditions, les phénomènes liés au fluage d'une part et au traînage visqueux d'autre part sont négligeables[2]. La contrainte d'écoulement est considérée comme la résultante de deux types de mécanismes:

- les premiers, thermiquement activés, correspondent au franchissement par les dislocations d'obstacles à faible rayon d'action (inférieur à une dizaine de distances inter-atomiques);

- les seconds, athermiques, soit sont intrinsèquement indépendants de la vitesse de déformation et de la température, soit ont des énergies et des distances d'activation trop importantes pous que ces facteurs aient un effet notable.

Il résulte de cette hypothèse que la contrainte d'écoulement est la somme de deux composantes: la contrainte interne $\tau_{a}$ correspond principalement à l'influence des défauts microstructuraux et la contrainte effective $\tau^{*}$ traduit les effets de la vitesse de déformation $\dot{\gamma}$ et de la température $T$.

$$
\tau=\tau^{*}+\tau_{a}
$$

Les expressions retenues pour les deux composantes de la contrainte sont

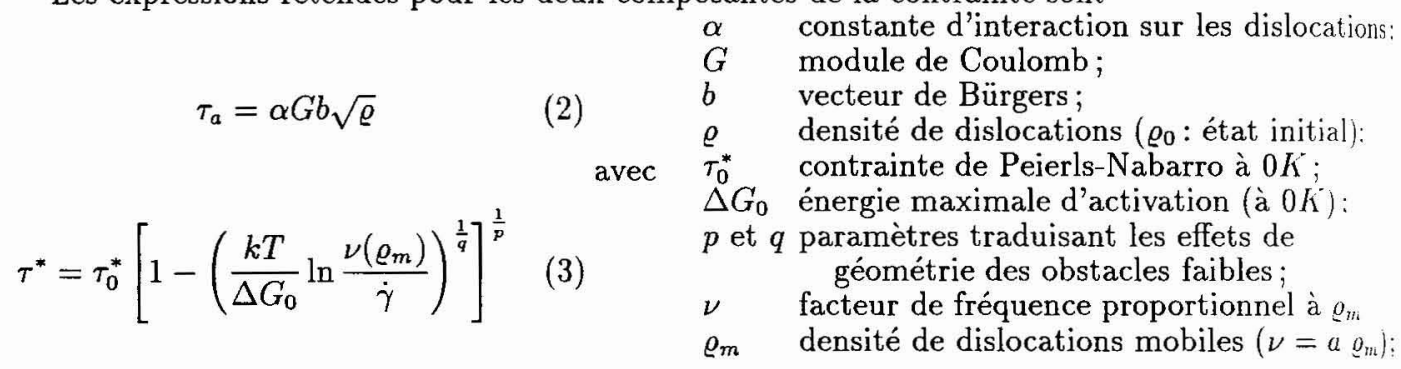

\subsection{Lois d'évolution}

La modélisation de l'évolution de la microstructure constitue un point essentiel. La densité de dislocations croît de manière quasiment linéaire avec la déformation au cours des premiers stades de l'écrouissage et ce, indépendamment de la température. Par la suite, on assiste à une recombinaison des dislocations que Klepaczko suppose proportionnelle à la probabilité de rencontre des dislocations donc à la densité de dislocations[1]. Nous reprenons ici cette démarche en adoptant une loi d'évolution du type:

$$
\frac{d \varrho}{d \gamma}=M_{I I}(\dot{\gamma})-k_{a}(\dot{\gamma}, T)\left(\varrho-\varrho_{0}\right)
$$

où $M_{I I}$ et $k_{a}$ désignent les coefficients respectivement de multiplication et d'annihilation des dislocations. Pour un chargement à vitesse constante, l'intégration de l'équation (4) conduit à l'expression de la densité de dislocations en fonction de la déformation qui peut être aisément inversée.

$$
\varrho=\varrho_{0}+\frac{M_{I I}}{k_{a}}\left(1-e^{-k_{a} \gamma}\right)
$$

En ce qui concerne la densité de dislocations mobiles, nous avons choisi la loi d'évolution suivante:

$$
\frac{\partial \varrho_{m}}{\partial \varrho}=\frac{\beta}{\varrho}
$$

où $\beta$ traduit la multiplication de dislocations mobiles et $\varrho_{m_{0}}$ la densité de dislocations mobiles initiale. Cette équation, une fois intégrée, donne:

$$
\varrho_{m}=\varrho_{m_{0}}+\beta \ln \frac{\varrho}{\varrho_{0}}
$$

\section{DISPOSITIF EXPERIMENTAL ET RESULTATS}

Les essais de torsion ont été effectués au moyen de Barres de Hopkinson en Torsion développé au CEA-DAM[6]. Ce dispositif permet de réaliser des torsions à vitesse constante ainsi que des snuls de vitesse dans un domaine compris entre $\dot{\gamma}=3 \cdot 10^{-4} \mathrm{~s}^{-1}$ et $\dot{\gamma}=3.10^{2} \mathrm{~s}^{-1}$. Le tableau 1 synthétise l'ensemble des essais réalisés. Ces essais ont servi d'une part à la détermination des constantes du matériau pour le modèle et d'autre part à la validation des hypothèses formulées. 
Tableau 1 : Ensemble des essais effectués.

\begin{tabular}{|l||c|c|c|c|}
\hline \hline$n^{\circ}$ & $\dot{\gamma}_{1}\left(s^{-1}\right)$ & $\gamma_{\text {saut }}(-)$ & $\dot{\gamma}_{2}\left(s^{-1}\right)$ & $\gamma_{\text {fin }}(-)$ \\
\cline { 2 - 5 } 1 & $3.10^{2}$ & 0,36 & $3.10^{2}$ & 0,72 \\
2 & $3 \cdot 10^{-4}$ & 1,20 & - & - \\
3 & $3 \cdot 10^{-4}$ & 0,05 & $3.10^{2}$ & 0,42 \\
4 & $3 \cdot 10^{-4}$ & 0,10 & $3 \cdot 10^{2}$ & 0,47 \\
5 & $3 \cdot 10^{-4}$ & 0,18 & $3 \cdot 10^{2}$ & 0,57 \\
6 & $3.10^{-4}$ & 0,36 & $3.10^{2}$ & 0,72 \\
7 & $1,5 \cdot 10^{-2}$ & 0,05 & $3.10^{2}$ & 0,42 \\
\hline
\end{tabular}

\begin{tabular}{|l||c|c|c|c|}
\hline \hline$n^{\circ}$ & $\dot{\gamma}_{1}\left(s^{-1}\right)$ & $\gamma_{\text {saut }}(-)$ & \multicolumn{1}{c|}{$\dot{\gamma}_{2}\left(s^{-1}\right)$} & $\gamma_{\text {fin }}(-)$ \\
\cline { 2 - 5 } 8 & $1,5 \cdot 10^{-2}$ & 0,12 & $3.10^{2}$ & 0,50 \\
9 & $1,5.10^{-2}$ & 0,21 & $3.10^{2}$ & 0,58 \\
10 & $3.10^{2}$ & 0,21 & $3.10^{-4}$ & 1,20 \\
11 & $3.10^{2}$ & 0,36 & $3.10^{-4}$ & 1,20 \\
12 & $3.10^{2}$ & 0,21 & $1,5.10^{-2}$ & 1,20 \\
13 & $3.10^{2}$ & 0,36 & $1,5.10^{-2}$ & 1,20 \\
& & & & \\
\hline
\end{tabular}

Les courbes "isothermes" obtenues après traitement des données sont les suivantes:
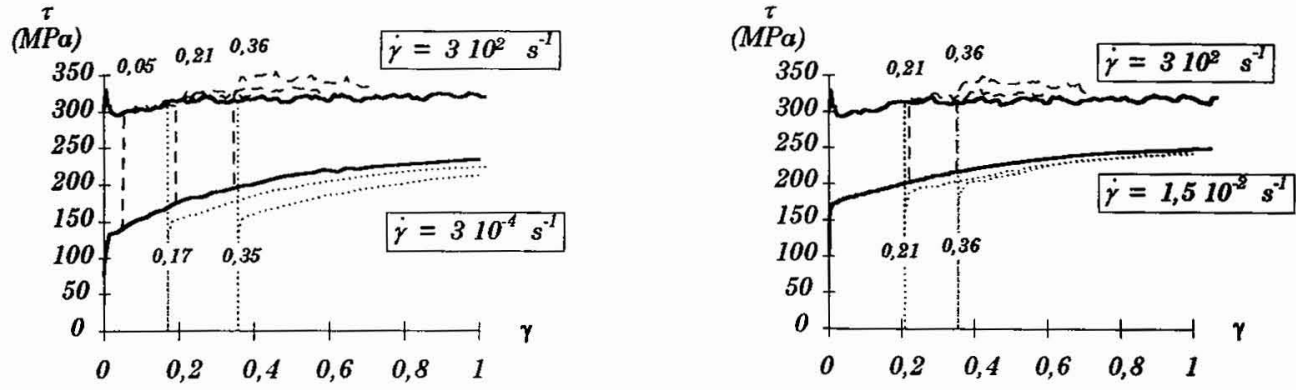

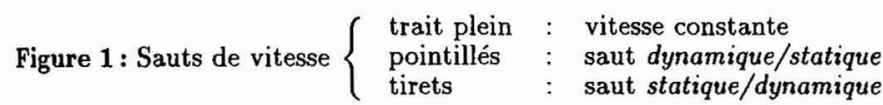

\section{DETERMINATION DES PARAMETRES}

\subsection{Hypothèses}

Afin de pouvoir déterminer les paramètres inconnus, nous sommes amenés à formuler plusieurs hypothèses. En ce qui concerne la contrainte interne nous avons supposé que:

H1 la contrainte effective est négligeable à $\dot{\gamma}=10^{-4} \mathrm{~s}^{-1}$ (nous sommes sur le plateau athermique[3, $7,8]$ ); cette hypothèse a été confirmée lors d'essais complémentaire effectués en compression à une vitesse constante de $\dot{\varepsilon}=10^{-5} \mathrm{~s}^{-1}$;

H2 le coefficient d'interaction $\alpha$ est constant tout au cours de la déformation et ce quelle que soit la vitesse de déformation; nous retenons la valeur théorique estimée par plusieurs auteurs $[9,10]$ : $\alpha=0,5$.

De plus, à partir des essais effectués, nous n'avons pu déterminer la forme des obstacles "thermiquement activés". Nous nous sommes appuyés sur les valeurs trouvées par Hoge et Mukherjee[11] à partir des travaux de Dorn[12] et reprises par Steinberg[13] :

H3

$$
p=1 \quad q=2 \quad \Delta G_{0}=0,62 \mathrm{eV}=10^{-19} \mathrm{~J} .
$$

\subsection{Contrainte interne}

Pour alléger les notations, les valeurs des fonctions de multiplication et d'annihilation des dislocations seront notées $M_{I I_{1}}$ et $k_{a_{1}}$ à $3.10^{-4} s-1, M_{I I_{2}}$ et $k_{a_{2}}$ à $1,5.10^{-2} s-1, M_{I I_{3}}$ et $k_{a_{3}}$ à $3.10^{2} s-1$.

4.2.1. Valeurs sur le palier athermique

D'après H1 la valeur de la contrainte à $3 \cdot 10^{-4} \mathrm{~s}^{-1}$ est celle de la contrainte interne.

$$
\tau=\tau_{a}=\alpha G b \sqrt{\varrho_{0}+\frac{M_{I I_{1}}}{k_{a_{1}}}\left(1-e^{-k_{a_{1}} \gamma}\right)}
$$

Par une méthode d'optimisation des moindres carrés nous trouvons ainsi :

$$
\begin{aligned}
\varrho_{0} & =1,25 \cdot 10^{14} \mathrm{~m}^{-2} \\
M_{I I_{1}} & =9,8 \cdot 10^{14} \mathrm{~m}^{-2} \\
k_{a_{1}} & =2,2
\end{aligned}
$$




\subsubsection{Valeurs à $3.10^{2} \mathrm{~s}^{-1}$}

D'après H3, la microstructure n'évoluant pas entre deux chargements, lors d'un saut de $3.10^{2}$ à $3.10^{-4} s^{-1}$, la densité de dislocations à la fin d'un chargement dynamique est identique à celle du début d'un chargement statique. L'intégration de la loi d'évolution de la densité de dislocations conduit à :

avec

$$
\begin{gathered}
\varrho=\varrho_{0}+\frac{M_{I I_{1}}}{k_{a_{1}}}\left(1-e^{-k_{a_{1}}\left(\gamma-\gamma_{s a u t}\right)}\right)+\left(\varrho_{s a u t}-\varrho_{0}\right) e^{-k_{a_{1}}\left(\gamma-\gamma_{s a u t}\right)} \\
\varrho_{s a u t}=\varrho_{0}+\frac{M_{I I_{3}}}{k_{a_{3}}}\left(1-e^{-k_{a_{3}} \gamma_{s a u t}}\right)
\end{gathered}
$$

Comme après le saut nous sommes sur le plateau athermique, la densité de dislocations $\varrho_{s a u t}$ présente au sein du matériau juste après le saut peut être déterminée par la relation issue de l'équation (2): $\varrho=\left(\frac{\tau}{\alpha G b}\right)^{2}$ où $\tau$ désigne la nouvelle limite d'élasticité. En effectuant des sauts aux mêmes vitesses de déformation mais à des taux $\gamma_{s a u t}$ différents, on aboutit à plusieurs couples $\left(\gamma_{s a u t}, \varrho_{s a u t}\right)$. On parvient ainsi à évaluer les coefficients $M_{I_{3}}$ et $k_{a_{3}}$. Les résultats des essais sont les suivants:

Tableau 2 : Détermination de $M_{I I_{3}}$ et $k_{a_{3}}$ à partir des sauts de $3 \cdot 10^{2}$ à $3 \cdot 10^{-4} s^{-1}$.

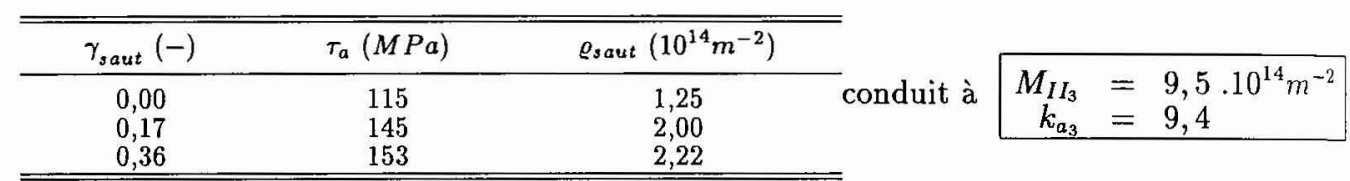

\subsubsection{Valeurs à $1,5.10^{-2} \mathrm{~s}^{-1}$}

La phase de détermination à $\dot{\gamma}=1,5 \cdot 10^{-2} s^{-1}$ nécessite une nouvelle hypothèse qui stipule que. rapidement après le saut de vitesse statique/dynamique, la densité de dislocations mobiles $\varrho_{m}$ atteint une valeur limite, conduisant ainsi à une saturation de la contrainte effective. Lors d'un saut de $1,5.10^{-2}$ à $3.10^{2} \mathrm{~s}^{-1}$, l'écart de contrainte entre la partie dynamique de la courbe de saut et la courbe à vitesse constante est alors dû uniquement à une variation de contrainte interne.

Nous déterminons des couples $\left(\tau_{a}, \gamma_{s a u t}\right)$ et donc de nouveaux doublets $\left(\varrho, \gamma_{s a u t}\right)$ qui permettent d'aboutir à :

$$
\begin{aligned}
M_{I I_{2}} & =9,7 \cdot 10^{14} \mathrm{~m}^{-2} \\
k_{a_{2}} & =3,1
\end{aligned}
$$

\subsection{Contrainte effective}

\section{$\stackrel{\tau}{\mathcal{\tau}}(\mathbf{P} a)$}

Nous avons adopté une géométrie d'obstacle proposée dans la littérature. Cependant la contrainte de Peierls-Nabarro $\tau_{0}^{*}$ et le facteur de fréquence $\nu=a * \varrho_{m}$ demeurent à déterminer.

\subsubsection{Contrainte de saturation $\tau_{0}^{*}$}

La contrainte effective à $300 \mathrm{~s}^{-1}$ est déterminée en ôtant la composante interne à la valeur mesurée de la contrainte. Dans le cas du tantale, nous constatons que la contrainte effective tend rapidement vers une valeur asymptotique. Cela signifie que la densité de dislocations mobiles $\varrho_{m}$ atteint un état saturé (figure 2).

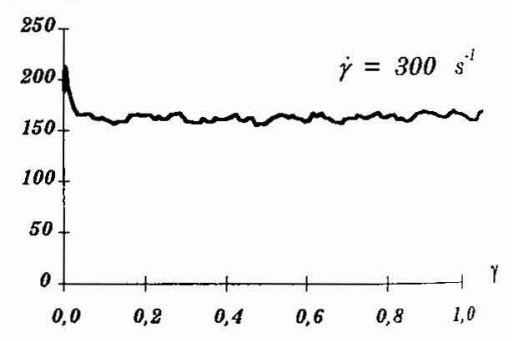

En première approximation, en négligeant l'effet de la vitesse de déformation sur le facteur de fréquence, on aboutit à l'expression[14]:

$$
\Delta G\left(\tau^{*}\right)=-k T^{2}\left(\frac{\partial \tau^{*}}{\partial T}\right)_{\dot{\gamma}}\left(\frac{\partial \ln \dot{\gamma}}{\partial \tau^{*}}\right)_{T}
$$

Lors d'essais successifs sur la même éprouvette, nous avons mesuré un coefficient d'adoucissement dû à la température de $\left(\frac{\partial \tau^{*}}{\partial T}\right)_{\dot{\gamma}=3.10^{2}}=-0,475 M P a . K^{-1}$. La sensibilité instantanée déterminée à partir des différents sauts est $\left(\frac{\partial \tau^{\circ}}{\partial \ln \dot{\gamma}}\right)_{T=300 K}=12 M P a$ ce qui, en estimant la contrainte effective de 
saturation à $162 M P a$ donne, en accord avec la littérature[11, 13, 14]:

$$
\Delta G_{\mid 162 M P_{a}}=0,29 \mathrm{eV}=4,710^{-20} \mathrm{~J} .
$$

L'expression de l'énergie d'activation retenue $\Delta G=\Delta G_{0}\left[1-\left(\frac{\tau^{*}}{\tau_{0}^{*}}\right)^{p}\right]^{q}=0,62\left[1-\left(\frac{\tau^{*}}{\tau_{0}^{*}}\right)^{2}\right]$ conduit à

$$
\tau_{0}^{*}=515 \mathrm{MPa}
$$

\subsubsection{Facteur de fréquence}

A partir de la courbe de la contrainte effective (figure 2), et en estimant le facteur $a$ de proportionnalité entre le facteur de fréquence et la densité de dislocations mobiles à $a=8,2.10^{-6} \mathrm{~m}^{2} \mathrm{~s}^{-1}[6]$, nous avons optimisé les coefficients de l'équation (7) pour trouver :

$$
\begin{aligned}
\beta & =6,2 \cdot 10^{12} m^{-2} \\
\varrho_{m_{0}} & =1,5 \cdot 10^{11} m^{-2}
\end{aligned}
$$

\section{MODELISATION}

Les paramètres du modèle ont été affinés globalement par un programme d'optimisation sur l'ensemble des essais. Les modifications n'ont pas été très notables.

Tableau 3: Coefficients du tantale d'étude.

\begin{tabular}{cccccccc}
\hline$\alpha(-)$ & $G(G P a)$ & $b(n m)$ & $\varrho_{0}\left(m^{-2}\right)$ & $M_{I I}\left(m^{-2}\right)$ & $k_{a_{1}}(-)$ & $k_{a_{2}}(-)$ & $k_{a_{3}}(-)$ \\
0,5 & 72 & 0,286 & $1,25.10^{14}$ & $10^{15}$ & 2,1 & 3,1 & 4,1 \\
\hline \multicolumn{10}{c}{} \\
\hline$\Delta G_{0}(\mathrm{eV})$ & $p(-)$ & $q(-)$ & $\tau_{0}^{*}(\mathrm{MPa})$ & $a\left(m^{2} \cdot s^{-1}\right)$ & $\varrho_{m_{0}}\left(m^{-2}\right)$ & $\beta\left(m^{-2}\right)$ \\
0,62 & 1 & 2 & 490 & $8,2.10^{-6}$ & $5.10^{12}$ & $2.10^{13}$ \\
\hline
\end{tabular}

Avec le jeu de constantes du tableau 3 , nous obtenons les résultats suivants:
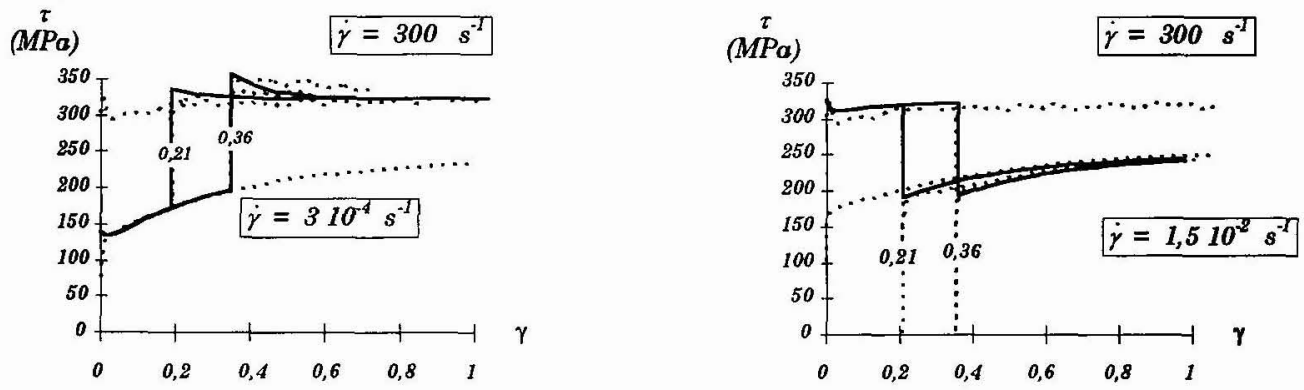

Figure 3 : Simulations de sauts de vitesse $\left\{\begin{array}{lll}\text { trait plein } & : & \text { simulation } \\ \text { pointillets } & \text { essai }\end{array}\right.$

\section{DISCUSSION}

Une étude parallèle menée sur le même tantale nous a montré que la limite d'élasticité inférieure croit de manière quasi linéaire en fonction du logarithme de la vitesse de déformation. Le volume d'activation relié au processus de viscoplasticité est très faible [14]:

$$
V^{*}=-\frac{d \Delta G}{d \tau^{*}} \approx k T \frac{\partial \ln \dot{\gamma}}{\partial \tau^{*}}=14 b^{3}=3,1.10^{-28} \mathrm{~m}^{-3}
$$

Il ressort de cette détermination que la multiplication de dislocations, très peu sensible à la vitesse de déformation, apparaît comme un processus athermique. Cette constatation conjuguée à la faiblesse du volume d'activation, nous laisse penser que le mécanisme prépondérent est une création à partir de sources de Frank-Read.

En revanche, l'annihilation des dislocations présente une très grande sensibilité à la vitesse de déformation. La densité de dislocations évolue vers une valeur de saturation $\varrho_{\text {sat }}$ qui diminue avec la vitesse de déformation.

Nous avons choisi une loi d'évolution de $\varrho_{m}$ qui, d'une part, présente de grandes variations dans les premiers stades de la déformation (lorsque $\varrho$ est faible) et, d'autre part, tend vers un état saturé 
lorsque la densité de dislocations totale atteint une valeur limite. Cette loi conduit à de très bonnes simulations pour les essais à vitesse constante ou les sauts dynamique/statique mais montre ses linites pour les sauts statique/dynamique car la variation modélisée de $\varrho_{m}$ est alors trop lente.

En dépit de cette restriction, il apparaît que le modèle retranscrit, à partir de l'analyse sur pen de points expérimentaux, plusieurs phénomènes observés.

- Tout d'abord, il est compatible avec la très forte sensibilité instantanée à la vitessc' de déformation.

- Le modèle parvient à restituer un écrouissage qui décroît avec la vitesse de déformation.

- Enfin, il retraduit des effets liés à l'histoire du chargement qui n'apparaissent qu'à uil srade avancé de la déformation. Ceci est justifié en examinant l'évolution de la microstructure o. Au tout début, la densité de dislocations suit une loi indépendante de la vitesse de défomation $\varrho \approx \varrho_{0}+M_{I I} \gamma$. Un saut de vitesse dans cette zone ne montre pas d'effet d'histoire. Cependant. au delà de $\gamma=0,2, \varrho$ tend vers une valeur asymptotique $\varrho_{s a t}=\varrho_{0}+\frac{M_{I I}}{k_{a}(\dot{\gamma})}$ qui distigue deux chargements à des vitesses différentes et rend "visible" les effets d'histoire.

\section{CONCLUSION}

Il a été montré dans cet article qu'à partir de quelques essais et principalement des sauts vers le palier athermique, il est possible de séparer simplement les contributions des phénomènes "athermiques" et "thermiquement activés". Ce modèle se situe à la frontière entre d'un côtr des études microstructurales plus fines qui permettent de dissocier chaque phénomène (influence de la texture, des éléments interstitiels, de la taille de grains...) et d'approfondir les hypothèses formulées dans le paragraphe 4.1, et d'un autre côté des modèles plus "classiques" qui ne considèrent que des variables macroscopiques (déformation, travail emmagasiné) par rapport auxquels le présent modèle offre une richesse de simulation pour un coût en calcul relativement faible.

\section{Remerciements}

Les auteurs tiennent à remercier MM. Gilles Naulin (CEA Valduc) et Alain Matagne (CEA Bruyèresle-Châtel) pour leur aide précieuse.

\section{Références}

[1] Klepaczko J.R., Mat. Sc. \& Eng., 18, (1975), p 121.

[2] Klahn D, Mukherjee A.K. et Dorn J.E., Strain rate effects, 2nd int. conf. on the Strength of Metals and Alloys, Asilomar, 3, (1970), p 951.

[3] Frost H.J. et Ashby M.F., Deformation-mechanism maps : the plasticity and creep of metals and ceramics, Pergamon Press, (1982), p 30.

[4] Klepaczko J.R.,"Modeling of structural evolution at medium and high strain-rates, f.c.c. and b.c.c. metals", Proc. 8th Risö Symp., Constitutive relations and their physical basis, (1987). p 387.

[5] Klepaczko J.R., Sasaki T. et Kurokawa T., Trans. Jap. Soc. of Aer. Sc., 36, nº113, (1993). p 170.

[6] Buy F.,"Etude expérimentale et modélisation du comportement plastique d'un tantale. Prise en compte de la vitesse de déformation et du trajet de chargement", th. Univ. Metz, (1996).

[7] Conrad H., J. of Metals, 16, (1964), p 582.

[8] de Fouquet J., "Mécanismes fondamentaux de la déformation à froid des métaux", Mise en forme des métaux et alliages, ed CNRS, (1976), p 61.

[9] Wasserbäch W., Mat. Sc. E Tech., 96,(1987), p 167.

[10] Saada G., Acta Met., 8, (1960), p 841.

[11] Hoge K.G. et Mukherjee A.K, J. of Mat. Sc., 12, (1977), p 1666.

[12] Dorn J.E. et Rajnak S., Trans AIME, 230, (1964), p 1052.

[13] Steinberg D.J., Equation of state and strength properties of selected materials, Lawrence Livermore National Laboratory, UCRL-MA-106439, (1996).

[14] Arsenault R.J., Acta Met., 14, (1966), p 831. 